\title{
Comparing Calculus Communication across Static and Dynamic Environments Using a Multimodal Approach
}

\author{
Oi-Lam Ng ${ }^{1,2}$
}

Published online: 4 April 2016

(C) Springer International Publishing 2016

\begin{abstract}
In this article, a thinking-as-communicating approach is used to analyse calculus students' thinking in two environments. The first is a 'static' environment in the sense of static visual representations, such as those found in textbook diagrams, while the second is a dynamic environment as exploited by the use of dynamic geometry environments (DGEs). The purpose of the article is to compare calculus students' communication as it is facilitated by each of these two environments, and to explore the role of paper- and digital-mediated representations for positioning certain ways of thinking about calculus. The analysis provides evidence that the participants employed different modes of communication utterances, gestures and touchscreen-dragging - and they communicated about fundamental calculus ideas differently when prompted by different types of representations. The study presents implications for teaching dynamic aspects of functions and calculus, and argues for a multimodal view of communication to capture the use of gestures and dragging for communicating dynamic and temporal mathematical relationships.
\end{abstract}

Keywords Thinking-as-communicating $\cdot$ High school calculus · Dynamic geometry environments · Textbook diagrams · Touchscreen-dragging

\section{Introduction}

Studies have shown that calculus students have significant difficulties connecting algebraic with graphical representations of calculus concepts and in dealing with simultaneous change of variables in calculus relationships (Tall and Vinner 1981; Tall 1986;

Oi-Lam Ng

oilamn@sfu.ca

1 Simon Fraser University, Burnaby, BC, Canada

2 Present address: The Chinese University of Hong Kong, Shatin, NT, Hong Kong SAR, The People's Republic of China 
Graham and Ferrini-Mundy 1989; Thompson 1994; Ubuz 2007). Within the North American curriculum, students are seldom given opportunities to work with covariation and functions in a dynamic sense during their pre-calculus years. Traditional methods for teaching functions and their transformations are inadequate, as functions are commonly only manipulated algebraically at the object level without making use of the idea of continuous change. The tendency to think of functions and graphs as static objects, rather than as dynamic processes, may contribute to their struggles in the learning of calculus.

Classic studies have offered insights into obstacles for learning various calculus concepts, such as graphical interpretations of the derivative, limit, rate of change, differentiation and the fundamental theorem of calculus (FTC). Tall and Vinner (1981) traced students' difficulties in the study of limits and continuity to a dichotomy between dynamic and static notions of the concepts. They characterized the problem as students having non-cohering concept images and concept definitions. Studies have shown that a dynamic limit definition, which includes a definite feeling of motion, has a strong influence on students (Williams 1991; Tall 1980). Even after students are exposed to formal limit instruction, they continue to hold dynamic views of limit.

These studies all contend that a dynamic conception is both easy to grasp and natural to develop for students. Graham and Ferrini-Mundy (1989) approached the problem via students' representation of limits, showing that students' algebraic understanding of limits is independent of their graphical understanding. When students were asked to evaluate limits of the form $\lim _{x \rightarrow a} f(x)$, they are quite successful, but when asked for a geometric interpretation, students showed very little understanding. In one interview, a student explained that limit problems were simply functions to be evaluated and that the graph cannot help them find an answer.

Thompson (1994) found that visual understanding of the simultaneous change of all three variables, $x, f(x)$ and $\int_{a}^{x} f(t) d t$ in the FTC to be a challenge for students. Similarly, a number of studies reported students' difficulties in creating a graphical representation of a function's rate of change function (Tall 1986; Ubuz 2007). Berry and Nyman (2003) confirmed students' algebraic-symbolic view of calculus and the fact that they found it difficult to make connections between the graph of a derived function and that of the function itself. Furthermore, they indicated that students' thinking about the links between the graphs of a function and its derived function was enhanced by asking students to 'walk' these graphs as if they were displacement-time graphs. Their study suggests that these tasks help students to extend their understanding of calculus concepts from a symbolic representation to a graphical representation - and to what they termed a 'physical feel'.

This brief review of some classic studies on students' difficulties in the learning of calculus reveals that a split exists between graphical and algebraic representations, and that students have difficulties working robustly with dynamic elements of calculus beyond a physical and intuitive level. While students still struggle with calculus, more recent studies have shown instances of positive effects on calculus learning made possible by technology. In particular, the introduction of dynamic geometry environments (DGEs) has given rise to new ways of doing and representing calculus (see sub-section "The Learning of Calculus in Dynamic Environments" for a fuller review). DGE capabilities fill the gap among numerical, algebraic and graphical representations of functions by producing a seemingly limitless table of values for an algebraic expression by means of the act of continuous dragging.

I hypothesised that students' communication of calculus ideas via DGEs (in particular, via touchscreen-based DGEs) would differ from that in a static environment in terms of 
how simultaneous change, graphs and dynamic properties of functions and relationships would be communicated. The study reported in this article investigated high school calculus students' communication across two different environments: the first is a 'static' one, in the sense of static visual representations as those found in textbook diagrams, while the second is a dynamic one, as exploited by the use of DGEs. The ability to make connections across representations and to work with dynamic elements of calculus lies at the core of developing calculus thinking. This article further contributes to current research on the role of DGEs for developing calculus thinking, by exploring the role of paper- and digitally-mediated representations in framing certain ways of thinking about calculus.

In the next two sub-sections, I review the ways that static visual representations (such as textbook diagrams) convey the idea of 'change' in the study of calculus, and then discuss literature that points towards certain positive effects of using DGEs for the learning of calculus. Then, in subsequent sections, I describe the communicational framework and specific methodological tools I used for studying calculus thinking, followed by detailed analyses of two pairs of students communicating across two environments. Finally, I conclude by discussing the role of paper- and digitallymediated representations for situating certain ways of thinking calculus, and some potential implications for teaching and learning.

\section{The Learning of Calculus Using Static Visual Representations}

Weber et al. (2012) conjectured that students' difficulties with function notation, their struggles to connect algebraic with graphical representations of functions, and their understanding of rate of change may explain their struggles to think about the derivative as a function. They also contend that traditional calculus textbooks do not support the thinking and learning of certain calculus concepts: "the definition of derivative, as it was found in the contemporary calculus books [...], failed to convey mental imagery that would support students in constructing the derivative function" (p. 278). The insufficiency of conveying functions, covariation and continuous change in a dynamic sense may have contributed to students' difficulties. Below, I review a popular university calculus textbook in North America (Calculus by Stewart 2008), in particular some its conventions for conveying calculus relationships, in order to support this claim.

The diagrams shown in Fig. 1 introduce the idea of the derivative and the areaaccumulating functions which are central to the study of calculus. Colours and symbols are used to name mathematical objects. In Fig. 1a, the red line labelled $t$ is the tangent to the function $f$ at point $P$, while the blue line is the secant to the function $f$ that passes through the points $P$ and $Q$. In Fig. 1b, the shaded pink region illustrates the area under $y=f(t)$ between $t=a$ to $t=x$, which generates a function of $x$, denoted by $g(x)$, the areaaccumulating function.

Due to their static nature, both diagrams in Fig. 1 are captioned with words to convey change of the variables $h$ and $x$, as $h \rightarrow 0$ and as $x$ varies, respectively. Words such as "approaches" and "becomes" are used to introduce movement and a state of change in Fig. 1a: "Notice that as $x$ approaches $a, h$ approaches $0[\ldots]$ and so the expression for the slope of the tangent line in Definition 1 becomes $f^{\prime}(a)=\lim _{h \rightarrow 0} \frac{f(a+h)-f(a)}{h}$ " (Stewart 2008, p. 145).

The word "notice" in the caption seems an interesting choice of word, because the diagram is actually static, and thus it does not readily invite readers to "notice" 


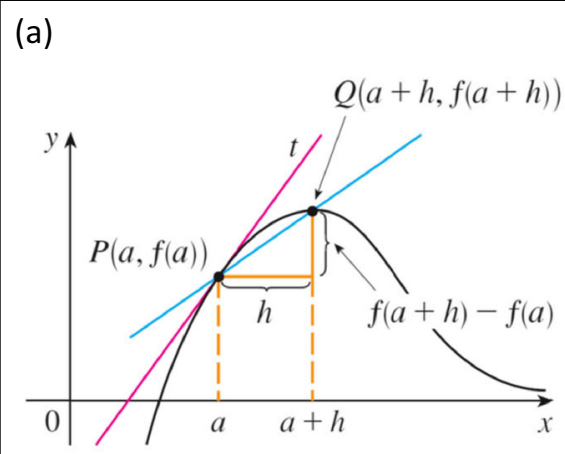

(b)

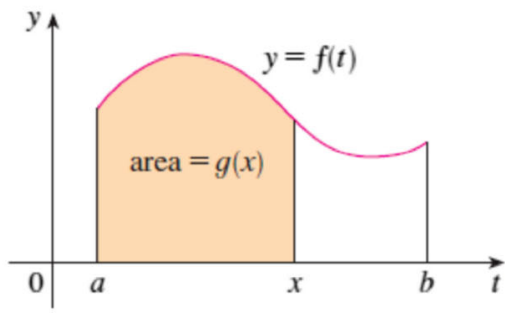

Fig. 1 a The relationship between secant and tangent to illustrate the definition of derivative. b The orange region illustrates the accumulating area, $g(x)$, as $x$ varies

anything changing in the diagram. Similarly, in Fig. 1b, the textbook diagram is accompanied by words to suggest the variation of $x$, as in: "If we then let $x$ vary, the number $\int_{a}^{x} f(t) d t$ also varies and defines a function of $x$ denoted by $g(x)$ " (p. 380). The conditional statement that begins with "if" suggests that there is a state of change, along with the verb "vary", which invites the readers to imagine the movement of $x$. Although both diagrams aim to communicate dynamic properties by means of static representations, it is the words that bear the dynamic quality of these properties.

Besides words, series of images are used to convey a sense of change in textbook diagrams. Fig. 2a shows a series of images illustrating a classic optimisation problem in differential calculus: maximize a rectangular area with a fixed perimeter. The variation of area with a fixed perimeter is implictly suggested - discretely - using three geometrical figures accompanied by numerical values of areas equal to $220,000 \mathrm{ft}^{2}, 700,000 \mathrm{ft}^{2}$ and $400,000 \mathrm{ft}^{2}$. Similarly, Fig. 2b shows a series of images depicting "the secant lines approaching the tangent line" (p. 82). It is interesting to note the plural form of "secant lines", because with a dynamic approach, there is only one secant line approaching the tangent line at a given point. Therefore, the idea of change is conveyed discretely and not continuously.

Point-wise and global trajectories are communicated using two graphs, one directly above the other, along with visual cues to suggest the same values on the $x$-axis. Figure 2c conveys the idea of plotting the slope of tangent at different points of the above graph, $y=f(x)=\sin (x)$, to obtain the graph of its derivative, $y=f^{\prime}(x)$. However, the dynamism of constructing of the derivative function is lost in the diagram, as seen in the caption, "by measuring slopes at points on the sine curve, we get strong visual evidence that the derivative of the sine function is the cosine function" (p. 172). "Measuring" the "slopes" at points (note "slopes" is plural too) on the sine curve implies that the process of obtaining the graph of $y=f^{\prime}(x)$ is a discrete one. Had the process been offered as a continuous dynamic one, the tangent slope (singular) would have been observed as $x$ varies instead.

\section{The Learning of Calculus in Dynamic Environments}

Evolution in digital technology has affected our thinking, learning and modes of interactions with mathematics. The invention of graphing and dynamic geometry 


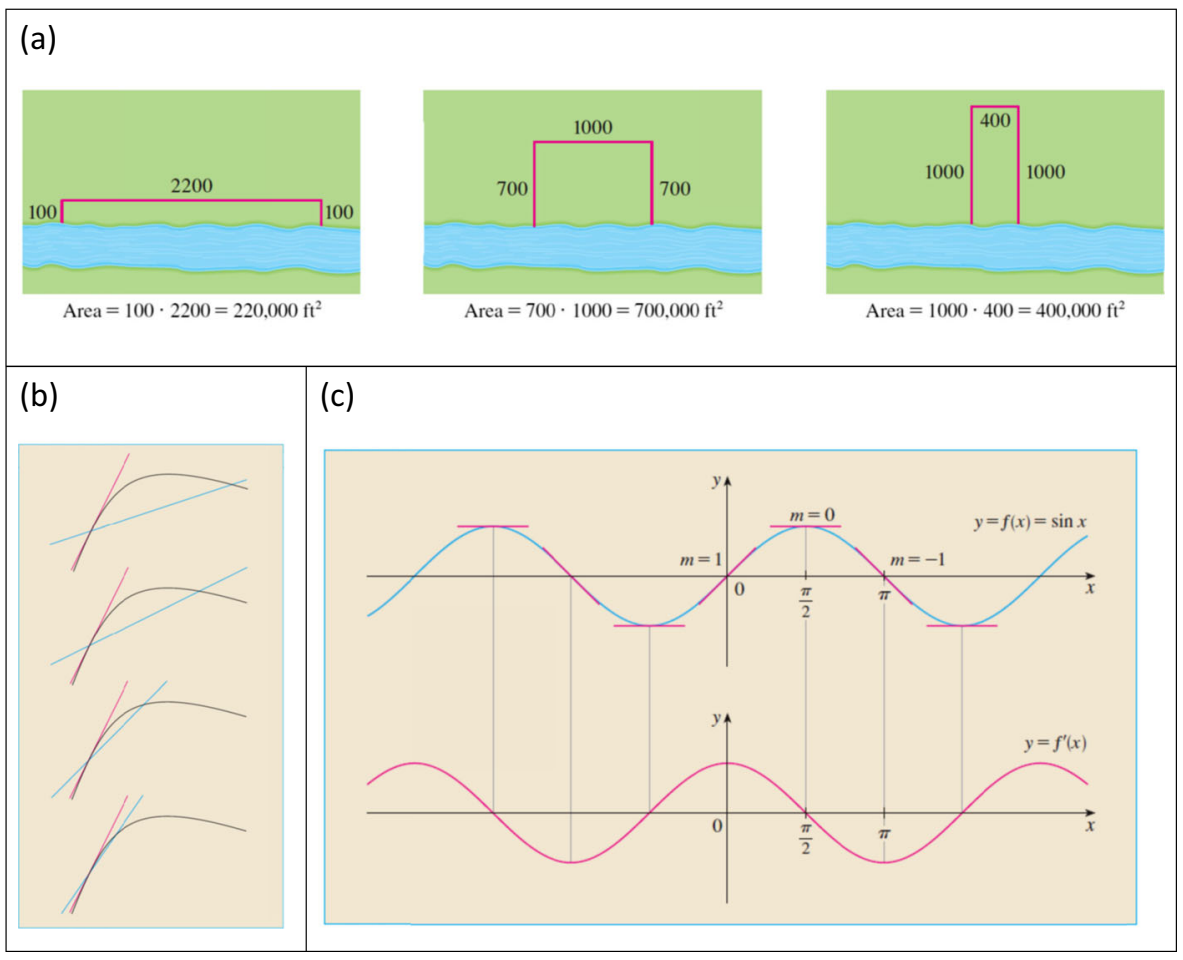

Fig. 2 a The variation of area using a series of images; b: "The secant lines approaching the tangent line" (p. 82) using a series of images; $\mathbf{c}$ : "By measuring slopes at points on the sine curve, we get strong visual evidence that the derivative of the sine function is the cosine function" (p. 172)

technologies has offered new ways of doing and representing mathematics. In particular, the dragging modality offered by DGEs enables users to interact with parameters in embodied ways and to observe change of functions dynamically. A growing number of research studies have focused upon the dynamic nature of DGEs and how this supports calculus thinking and learning. Research literature related to these ideas is reviewed in this sub-section.

A number of studies have investigated the learning of functions from a graphical point of view, using graphing and computer environments (Confrey and Smith 1994; Schwarz and Bruckheimer 1990; Cuoco 1994). These studies offer evidence of students overcoming difficulties with functions at the high school level. The digital environment enabled students to have control over a function by switching among representations and changing individual parameters. Falcade et al. (2007) showed how a DGE could help high school students grasp the notion of function; they focused on the affordances of the Trace tool as a semiotic mediator that could introduce the two-fold meaning of trajectory, both global and local (point-wise). Robutti and Ferrara (2002) introduced motion graphs via motion sensors that record displacement over time, concluding that the technology facilitated transitions between static and dynamic interpretations of the space-time graphs. Arzarello and Paola (2003) designed a teaching experiment involving students moving with respect to a motion sensor, where the goal was that the calculator should reproduce a graph that was as close as possible to the one drawn at the 
blackboard by the teacher. They argued that this embodied task using motion sensors is effective for introducing functions (and their first and second derivatives) within the same field of experience. By contrast, traditional teaching tasks for calculus introduced these aspects on separate occasions.

Distinguishing between the intuitive versus formal approaches to various topics in calculus, Ferrara et al. (2006) reviewed a number of studies using dynamic geometry technology for enhancing the teaching of derivatives and integrals. In one study, a DGE was used to plot a function along with its derivative dynamically on the same screen. As one student explained, "I never understood what it meant to say that the derivative of $\sin x$ is cosx until I saw it grow on the computer" (p. 261). As the student saw the derivative function "grow" on the screen, the covariation between tangent slope and derivative functions was mediated dynamically and continuously. Moreover, "They seem far more willing to discuss conceptual difficulties thrown up by the computer than they would difficulties in understanding a teacher's explanation" (p. 261).

Investigating how students understand rate of change has naturally led to the study of how students understand Riemann sums and the fundamental theorem of calculus (FTC) graphically. Thompson et al. (2013) emphasise an approach that allows students to explore variation and covariation in a technological environment, prior to leading up to the study of the FTC. They designed a digital environment that simulates the 'bottle problem', in which water accumulates in a bottle: the participants were asked to graph the volume of water in the bottle as a function of its height. The authors argue that this approach helps students to build a reflexive relationship between concepts of accumulation and rate of change, one which could only be possible with the use of technology.

Yerushalmy and Swidan (2012) used a semiotic lens through which to observe students' use of dynamic and multi-representation environment for learning the concept of accumulation graph. Their artifact was designed to support exploration using dynamic and multiple representations of an area-accumulation function. With an interface that allows interactive changes of parameters and direct manipulation of graphic objects, the graph of the area-accumulation function can be drawn simultaneously, directly below the given function. They found that the zeroes of the accumulation graph and the use of colour coding for positive and negative areas served pivotal roles in the process of semiotic mediation.

Hong and Thomas (2013) examined the design of a curriculum where students used digital technology to develop a more balanced dual view of calculus ideas as both process and concept. Their results called for a teaching approach incorporating frequent use of dynamic geometry technology and graphing calculators, in order to encourage versatile embodied and inter-representational thinking. Further, using a calculator to display both numerical and graphical representations and engagement with them can support students in constructing derived functions and the development of local or interval thinking.

\section{Theoretical Framework}

The notion that learning mathematics invovles building mathematical communicative competence is suitable for the current study because it establishes a strong link between mathematics learning and communication. The learning as participation perspective complements this view; it is a broader framework for conceptualising learning in its 
social dimensions (Lave and Wenger 1991; Wenger 1998). This perspective suggests that learning is located neither in the heads nor outside of the individual, but in the relationship between a person and a social world. Thus, learning is cognitive, social, discursive and also semiotic, because it involves thinking, signs, and tools.

Sfard's (2008) communicational framework is based upon the social dimensions of learning and highlights the communicative aspects of thinking and learning. It has roots in the work of Vygotsky (1978) and Wittgenstein (1953), who both claimed that speech and thought are inseparable. Sfard redefines thinking as an "individualised version of (interpersonal) communicat[ion]" (p. 81). Her term commognition underscores her claim that thinking (individual cognition) and interpersonal communication are manifestations of the same phenomenon. This perspective is offered as a way to avoid the quandaries facing paradigms that treat learning as acquisition: rather, mathematical learning is regarded as expanding one's mathematical communication or discourse.

In her book, Sfard proposes four features of the mathematical discourse: word use, visual mediators, routines and narratives. These features can be used to analyse mathematical thinking and changes in thinking. For the purpose of this paper, the first three features will be used for analysing the utterances, gestures and dragging in two pair's mathematical discourse. Word use is a main feature in mathematical discourse; it is "an-all important matter because [...] it is what the user is able to say about (and thus to see in) the world" (p. 133). In addition, as a student engages in a mathematical problem, her mathematical discourse is not limited to the vocabulary she uses. For example, her hand-drawn diagram and gestures can be taken as a form of visual mediator to complement word use.

Routines are meta-rules defining a discursive pattern that repeats itself in certain types of situations. In learning situations, teachers may use certain words or gestures repeatedly to model a discursive pattern, such as looking for patterns and what it means to be "the same". Also, if, for example, a student repeatedly uses her arm to signify slope when comparing slopes of different line segments, she is using gestures as a routine to look for what is "the same". The same can be said of the use of dragging to compare the slopes of tangent at different points of the function. Hence, gestures and dragging can be taken both as a routine for defining a discursive pattern and as a visual mediator or multimodal feature of the students' discourse. This communication can be interpersonal when it is directed to another student or intrapersonal when it is directed to oneself.

Furthermore, Sfard (2009) explains that language and gestures should not be counter-posed to one another, since language is any symbolic system used in communication, and gestures are "the actual communication" (p. 194) that is visually mediated. Instead, she suggests that the proper verbal counterpart of gesture would be an utterance, a communicational act that is audio-mediated. According to Sfard, utterances and gestures inhabit different modalities that serve different functions in the commognitive process. Recursivity is a linguistic feature in mathematical discourse offered by utterances. The unlimited possibility to expand linguistically allows human to work in meta-discourse, or thinking about thinking.

On the other hand, gestural communication ensures all interlocutors "speak about the same mathematical object" (p. 197). Gestures are essential for effective mathematical communication: 'Using gestures to make interlocutors' realizing procedures public is an effective way to help all the participants to interpret mathematical signifiers in the same way and thus to play with the same objects" (p. 198). Gestures can be realised 
actually when the signifier is present, or virtually when the signifier is imagined. Sfard illustrates how a student uses "cutting", "splitting" and "slicing" gestures to realise the signifier "fraction". Since these gestures were performed in the air, where the signifier "fraction" is imagined, they provide an instance of virtual realization. Therefore, the same signifier "fraction" may be realised differently with different kinds of gesture or word use.

\section{Temporality, Gestures and DGEs}

Sfard (2008) argues that mathematics could not progress as a discipline without the process of objectifying actions into nouns. On the other hand, she cautions us that removing the temporality and context of processes hides important details. Although Sfard considers gestures and diagrams as different forms of visual mediators, her view of visual mediation does not distinguish between the static and the dynamic. For example, in the case of diagrams, the mediation by a hand-drawn diagram is different from that offered by a textbook diagram like the ones in sub-section "The Learning of Calculus Using Static Visual Representations", because temporality is captured in the act of drawing by hand. This is also true for gestures - and even more so for DGEs which readily mediate temporality and dynamism. This may explain why Sfard cautions against the removal of temporality in the development of mathematical discourse.

The temporal functions of gestures have not been widely examined in gesture studies. Leading gesture specialist David McNeil's (1992) categorization of gestures (into deictic, iconic, metaphoric, and beat) broadly characterizes the functional range served by gestures. For example, deictic gestures serve as pointing means, while metaphoric gestures serve to represent the mathematical objects themselves. Although useful for identifying the general functions of gestures, these categories also do not distinguish between the static and dynamic nature of gestures, in particular, when gestures are used to convey temporal relationship.

For example, when a person makes a metaphoric gesture to realize the signifier, $a$ linear function, it could be of static nature, with the arm or hand enacting the function, or of dynamic nature, with the hand or finger tracing the motion of the function's path. In the latter case, the gestures communicate temporal relationships of the linear function as opposed to the offering the shape of the linear function statically. A few studies have shown that temporality can be evoked by the use of dynamic visual mediators like gestures (Ng and Sinclair 2013; Sinclair and Gol Tabaghi 2010), especially in the study of calculus (Núñez 2006). These studies point to the dynamic and temporal aspects of mathematicians' thinking; they also reveal that mobile hand movements are important features of this type of mathematical thinking.

In relation to the role of DGEs for evoking temporality, DGEs enable learners to observe and manipulate visual objects that are moving and changing over time. Because of its dynamic nature, visual mediation via DGEs is significantly different from that supported by textbook diagrams. Static visual representations evoke images of static mathematical objects (such as triangles) or artifacts (such as the number line). In contrast, DGEs may evoke mathematical relationships and properties due to its potential to depict an invariant property of a mathematical object continuously. For example, a student may realise new mathematical properties by dragging a vertex of a triangle or a point on the number line dynamically. 
With respect to the study of calculus, concepts like the graphs of derivative functions can be evoked readily on a dynamic sketch by utilising the Dragging and Trace tools. Sinclair and Yurita (2008) drew on Sfard's communicational approach to investigate the impact of using DGEs on mathematical thinking by identifying changes in the teacher's discourse in a grade ten geometry class. They found significant differences in the ways that the teacher talked about geometric objects, when moving from a static to a dynamic environment. For example, he no longer relied on comparing a given static shape with a definition, but began to use the Dragging tool to show whether properties of a given quadrilateral can be 'broken'. Their study shows how DGEs may change the way teachers use visual artifacts and geometric reasoning, as well as speak about them.

In a similar way, my study used Sfard's communicational framework to examine changes in the mathematical discourse as students moved from one type of environment to another. In my comparison, I highlight the roles of textbook diagrams (static environment) and touchscreen-based DGEs (dynamic environment) for facilitating certain ways of talking (thinking) about calculus. I also take up gestures as a dynamic visual mediator for conveying temporal relationships. In other words, I use a combination of utterances, gestures and dragging routines on a touchscreen-based DGE to study the students' multimodal mathematical discourse. Furthermore, I extend Sfard's communicational theory by distinguishing between dynamic and static visual mediators, in an effort to 'bring back' temporality into mathematical discourse.

\section{Methods}

The study reported here was part of a larger research project aimed at examining patterns of high school students' communication about calculus as they interacted with touchscreenbased DGEs in pairs. In this article, I focus on the data concerning the participants' communication prompted by two different types of environments (static and dynamic). The study took place at the end of the school year, in the participants' regular calculus classroom during school hours, but not during an actual math class. At the time of study, the participants had just completed a year-long AP Calculus course, where key concepts were taught using a class set of touchscreen-based DGEs. Therefore, at the time of study, the participants were experienced with exploring and discussing calculus concepts through geometrical, dynamic sketches in pairs. These concepts included the definition of a derivative, derivative functions, related rates and the fundamental theorem of calculus. This particular setting allowed me to compare the participants' features of mathematical discourse about the same calculus concepts, but facilitated by different environments.

Two pairs of my grade 12 (age 17 to 18) students participated in the study: Ana and Tammy had been regular classroom partners, and Melissa and Yee had sat in proximity to each other, but were not regular classroom partners. These pairings were intended to foster the kinds of student-pair communication that would occur in the students' regular calculus classroom. The pairs were asked to discuss twelve different diagrams presented on the iPads - seven textbook diagrams shown in.pdf form and then five dynamic diagrams presented using iPad-based DGE application, SketchExplorer (Jackiw 2011). The seven textbook diagrams were taken from students' regular calculus textbook (Stewart 2008), and the five dynamic sketches were minimally adapted from the ones that the students had used in class during the school year. 
For the purpose of comparing patterns of communication, seven of the textbook diagrams had a corresponding dynamic sketch that involved the same target concept (for a list of target concepts, see Table 1). The order of presenting the diagrams to the participants was chronological, as they had been taught in the participants' regular classroom. Also, the choice of presenting the seven textbook diagrams before the dynamic sketches was based on my hypothesis that the students' discourse about the textbook diagrams would not have a significant impact on their later discourse about the DGEs (as opposed to the reverse order). I gave the participants the instructions: "discuss what you see, what concepts are involved" and then turned on the camera located in front of and facing the student-pairs and left the room, until the students had finished talking about all the diagrams. Each student-pair took around 25 min to complete the task.

As it was necessary for this study to capture student pairs' utterances, gestures and dragging actions in different environments, special decisions were made around the transcribing of data and its organisation. Unlike conventional transcripts which inform only "what was said", I included "what was done" in the transcript, in order to record "who gestured what" and "who dragged what". Snapshots of certain gesturing and dragging actions were taken and included in the transcripts. As a transcript convention, certain parts of the transcript were underlined and italicised to record which words were spoken while a dragging and gesturing action was being simultaneously performed by one of the students. My rationale was to legitimise each of the three modes of communication: speaking, gesturing and dragging as significant modes of communication for the students.

Besides studying each mode in isolation, I also studied the interplay between them for better understanding the participant pairs' patterns of discourse. This was achieved by attending to turn-taking; instances of simultaneous speaking, dragging and gesturing actions (either by the same person or by different persons); dragging or gesturing actions without accompanying speech. The list below summarises the units of analysis in the study.

1. Particular words or phrases that were used repeatedly.

2. Particular dragging and gesturing actions that were used repeatedly.

3. Turn-taking: modes of communications (speaking, dragging, gesturing) when a new turn began.

Table 1 Calculus topics discussed by the participants in the study

\begin{tabular}{lll}
$\begin{array}{l}\text { Calculus topics discussed by } \\
\text { the participants in the study }\end{array}$ & $\begin{array}{l}\text { Textbook diagrams used in the } \\
\text { study and included in this paper }\end{array}$ & $\begin{array}{l}\text { Dynamic sketches used in the study and } \\
\text { their screenshots included in this paper }\end{array}$ \\
\hline $\begin{array}{l}\text { Definition of a derivative } \\
\begin{array}{l}\text { Derivative functions } \\
\text { Related rates }\end{array}\end{array}$ & $\begin{array}{l}\text { Figure 1a } \\
\text { Figure 2c }\end{array}$ & Figure 3a to d \\
$\begin{array}{l}\text { Optimization problems } \\
\begin{array}{l}\text { Mean value Theorem } \\
\text { Newton's Method }\end{array}\end{array}$ & Figure 2a & $\begin{array}{l}\text { Figure } 5 \\
\text { Corresponding dynamic sketch not used }\end{array}$ \\
\hline
\end{tabular}


4. Particular words that were used simultaneously with dragging or gesturing actions by one or two persons.

5. Dragging or gesturing without accompanying speech by one or two persons.

6. Recurrent sentence structures within a certain time span.

Besides comparing the list mentioned above to characterise students' patterns of discourse in different environments, I also compared the mathematical content communicated by the students, especially as it pertained to dynamic elements of calculus. To achieve this, the data was analysed in terms of the mathematical processes (NCTM 2000) demonstrated by the participants. These processes, which include reasoning, conjecturing, modelling, connecting and proofing, were intended to guide instructional programs by describing what teaching and learning should look like when attaining a certain learning outcome.

As the data collected was multimodal, two kinds of analyses, synchronic and diachronic, were carried out. According to Arzarello (2006), both are needed to understand semiotic activity fully. Synchronic analysis enables the study of relationship among different semiotic sets activated simultaneously. Diachronic analysis studies the same phenomenon in successive moments. The use of synchronic and diachronic analyses is suitable for the aims of the study: a synchronic lens is used to analyse the interrelationships among different modes of communication used by participants when engaging in calculus communication. On the other hand, a diachronic lens is used to investigate how the use of speech, gestures and dragging, change over time. Using Sfard's framework, such diachronic analysis enables the study to address changes in mathematical discourse as changes in mathematical thinking.

\section{Design of Sketches}

My study aims to support student communication pertaining to dynamic calculus relationships and to foster multiple representations of calculus. With this aim, the sketches used in the study were designed to highlight dynamic aspects of calculus, to exploit touchscreen dragging and to connect algebraic, geometrical and numerical representations of the calculus. To examine the role of touchscreen-dragging in mathematical thinking, the iPad application, SketchExplorer (Jackiw 2011), was used to present the sketches that I had originally designed using the computer program Geometer's Sketchpad (Jackiw 2001).

The sketch described below was used in the study to convey the definition of a derivative. It mainly features two functionalities offered by Geometer's Sketchpad: the Hide/Show button and the Dragging tool. These functionalities have the potential to evoke mathematical relationships that would have been difficult to capture in textbook diagrams. For example, Fig. 3a shows the screen of a page in the sketch when the first button "show function" is activated. The capability to show or hide this function with the press of a button enhances the effect of seeing the function as a reified mathematical object.

The Dragging tool can be combined with the Hide/Show button to communicate the relationship between objects effectively. Figure $3 \mathrm{~b}$ shows the screen of a sketch when the "show tangent" and the "show function" buttons are both activated. As the user drags the point of tangency along the graph dynamically, the slope of the tangent at different points on the graph changes correspondingly. Performing this kind of 
dragging enables the user to attend to the variation in the tangent slope (see Arzarello et al. 2002). Furthermore, by assigning "show tangent" (a second button available in the sketch), the tangent line can be seen as another reified mathematical object. Yet it is a "child" object, one dependent upon the function, since dragging is restricted to points on the graph only.

Figure $3 \mathrm{c}$ and d illustrate how a dynamic sketch may connect symbolic, numerical, and geometrical representations of calculus concepts. Upon activating the "show secant line" button in Fig. 3c, the green point can then be dragged along the graph and the corresponding numerical values of the secant slope is displayed. At the same time, the value of the secant slope is represented geometrically by two triangles (conveying rise and run). Therefore, dragging actions produce a simultaneous change to the numerical value, the rise and the run of the slope triangles. Finally, the last button, when activated, shows the numerical value of the secant slope calculation (see Fig. 3d).

The simultaneous change of the values of $f(x+h)$ and $h$, as well as the continuous change of the rise/run triangle, provide a strong visual mediation connecting algebraic, numerical and geometrical representations of the definition of a derivative. As the user drags the point of tangency continuously along the graph, the numerical calculations of the secant slope and the rise/run triangles change dynamically as well. Meanwhile, the use of colour enhances the visual effect, since the same colour is assigned to the

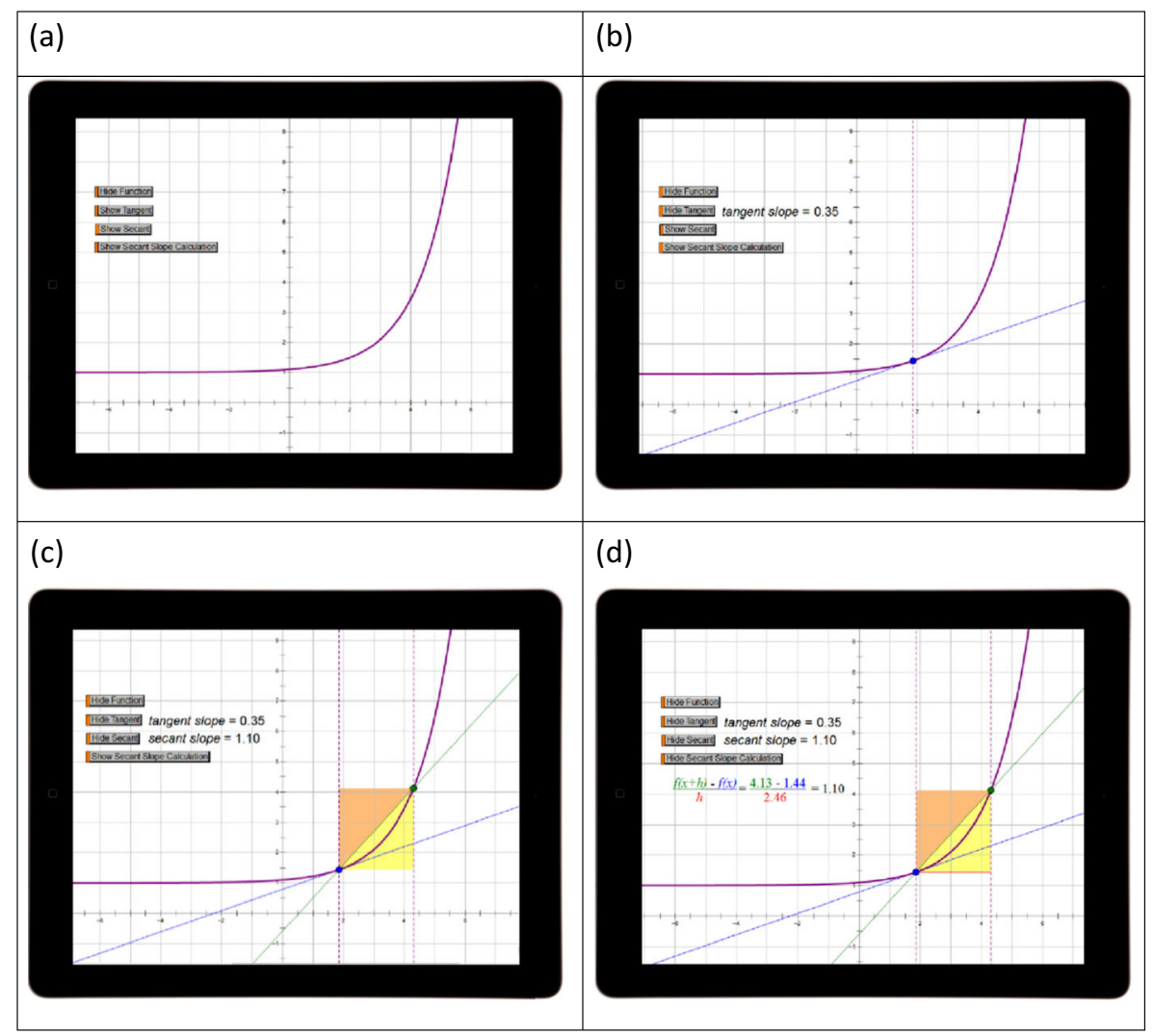

Fig. 3 a-d: Screenshots of a page in a dynamic sketch conveying the definition of a derivative 
mathematical object and its symbolic equivalent. For example, both $h$ and the "run" of the rise/run triangle are coloured red in Fig. $3 \mathrm{~d}$.

\section{Data Analysis}

In this section, I provide a four-part analysis of the participants' communication during the task, drawing on the communicational theoretical framework as overviewed in section "Theoretical Framework". Following the methods as described previously, I analysed the participant pairs' communication by attending to their patterns of discourse consisting of particular speech, gestures and dragging actions used by one or both participants synchronically and diachronically. In particular, two short episodes (five turns in each) are chosen from a 25-min discussion between the student pair, Ana and Tammy, and analysed in detail in sub-section "Comparing Gestures: Dragging as a Form of Gesturing".

It involves two episodes of Ana and Tammy's engagement with the task when they were given a textbook diagram and then a dynamic sketch related the definition of a derivative. Each episode begins with a transcript followed by an analysis. The episodes were chosen to characterise and contrast the non-linguistic modes of communication used by the student pair. Although the use of gestures was prevalent in Ana and Tammy's discourse with both textbook diagrams and with DGE, different types of gestures were observed in each situation, including the emergence of dragging as a form of gestural communication. This analysis grounds the work of further analysis in the sections to follow.

In sub-sections "Other Gestures Facilitated by a Static Environment" to "Communicating Variance and Invariance Through Dragging", I focus my analysis on my second participant pair, Melissa and Yee's, communication. In Ana and Tammy's communication, I analysed the student pair's engagement of the task on one particular topic and compared their communication across two environments. By contrast, I found Melissa and Yee's communication interesting in terms of particular features of communication relative to the mathematical ideas. Therefore, I summarise some key findings about this student pair's communication about various mathematical ideas in three sub-sections. In addition, I highlight particular words, gestures and dragging actions that were not observed in the previous analysis about Ana and Tammy.

\section{Comparing Gestures: Dragging as a form of Gesturing}

The following is a transcript ${ }^{1}$ of the first five turns between Ana (on the left) and Tammy's (on the right) discussion about the definition of a derivative when prompted by a textbook diagram Table 2 .

The transcript above shows that Ana and Tammy were mainly engaging in two kinds of mathematical processes, namely those of naming/identifying the mathematical objects shown in the textbook diagram. The students took turns to name the red line,

\footnotetext{
${ }^{1}$ Transcript conventions: Underlined transcripts denote words that were spoken while the speaker was gesturing. A comma (,) denotes short pauses, and a period (.) denotes longer pauses. The question mark (?) denotes high intonation.
} 
Table 2 Transcript and selected snapshots of Ana and Tammy's gestures while discussing a textbook diagram related to the definition of a derivative

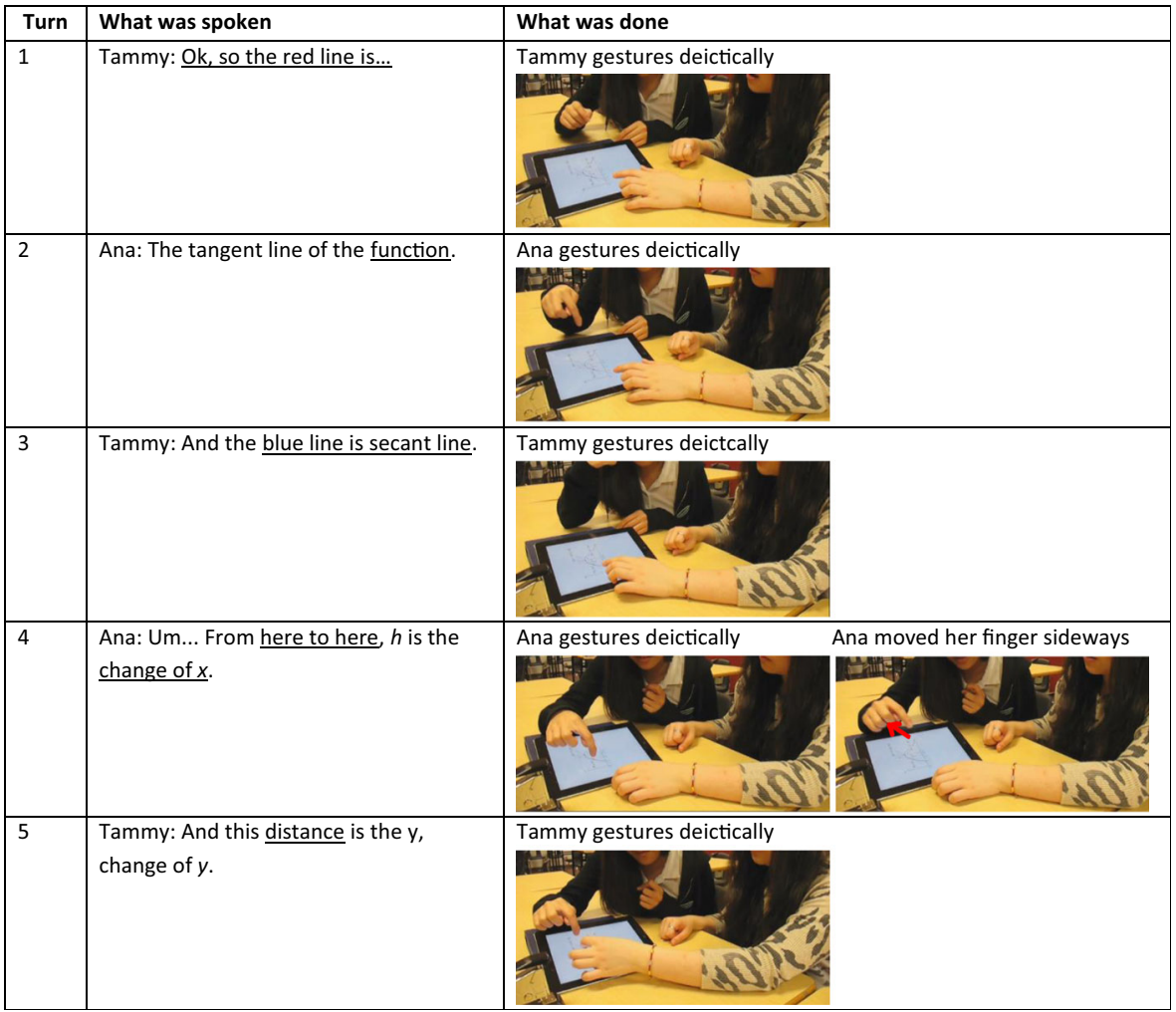

blue line, change of $x$, and change of $y$ respectively. The use of gestures was present, as the students identified each of the four mathematical objects during this part of their discussion. Of the six gestures shown in the transcript, five of them were deictic (pointing) gestures, while the speaker also referred to the particular objects in their utterances. For example, Tammy used her left index finger to point to the tangent line while she uttered "the red line is ..." (turn 1). Then, Ana completed Tammy's statement with "the tangent line of the function" (turn 2), accompanied by a similar pointing gesture with her right index finger pointing towards the tangent line.

Besides being observed frequently in their communication, these gestures also seemed significant in terms how they complemented utterances in the communication. With the use of deictic gestures like the ones found within the first five turns, word use was transformed: deictic words like "this distance" (turn 5) and "from here to here" (turn 4) appear in the students' utterances. Using deictic words, the speakers no longer needed to refer to the mathematical objects by describing them verbally, but instead they could use deictic gestures along with pronouns and locative nouns to replace the descriptions completely. Therefore, these gestures could significantly reduce the number of words that were needed to refer to the mathematical objects, as found in Tammy's "this distance is the change of $y$ " (turn 5) and Ana's "from here to here is 
the change of $x$ " (turn 4). As Sfard explains, gestures help ensure that the interlocutors speak about the same mathematical objects. As seen in this episode, Ana and Tammy were able to use a combination of utterances and gestures to communicate effectively about the mathematical objects. In particular, word use is transformed with the presence of deictic gestures.

The textbook diagram may have influenced Ana and Tammy's thinking about calculus. The students' mathematical discourse, as observed in their word use and gestures, reflected a static way of thinking about the definition of a derivative. The students resorted to the verb form "is" four times in this episode, each time followed by nouns "tangent line" (turn 2), "secant line" (turn 3), "change of $x$ " (turn 4) and "change of $y$ " (turn 6) respectively to name each of the visual mediators shown in the textbook diagram. These verb-noun combinations generated statements that are static in nature. During the first five turns, the only instance where dynamism was conveyed was when Ana moved her index finger while uttering "change of $x$ " in Turn 4 . When speaking about the "change of $x$ ", Ana moved her index finger laterally from left to right. It could be said that this gesture was deictic, pointing to the mathematical object, but it also served to enact the mathematical idea of "change of $x$ ", temporally.

Other than that, all of the gestures in this part of their discussion were for deictic purposes and static, as opposed to dynamic in the sense of conveying temporality in mathematics. These word use and gestures were highly relevant to the students' particular mathematical processes at the moment, of naming and stating mathematical objects, which are static in nature as well. The static mathematical objects evoked by the textbook diagram may have facilitated this form of communication.

Below, I analyse the transcript ${ }^{2}$ containing the first five turns between Ana and Tammy's discussion about a dynamic sketch relating to the definition of a derivative Table 3.

When the students initially opened the sketch, two buttons were already in the "show" position on the page - therefore, the graph of a parabola, $f(x)=x^{2}$ and its tangent line at a given point appeared on the sketch. As can be seen in the transcript, Ana and Tammy explored the dynamic sketch using the dragging modality. In the first exchange, Tammy's utterance, "tangent line is increasing" (turn 1) was accompanied by dragging the point of tangency from left to right (although technically it was the tangent slope that was increasing and not the tangent line). Following that, Ana seemed to be mirroring Tammy's utterance-dragging combination with "the tangent [slope of the] line is zero" (turn 4), while dragging the point of tangency back towards the vertex. These were two of five series of dragging actions observed that spanned up to five seconds within the first fourteen turns of their discussion with a dynamic sketch.

My analysis suggests that these dragging actions were not solely dragging, but were also gestural communications - to communicate the dynamic features and properties in the sketch it the very moment of dragging. Recall that in a static environment, Ana and Tammy frequently used static, deictic gestures to refer to different mathematical objects. By contrast, these static, deictic gestures were not observed in the dynamic environment. Instead, the students' gestures were blended within their dragging actions as they spoke about the change of the tangent slope.

\footnotetext{
${ }^{2}$ Transcript convention: Italicised parts of the transcript denote words that were spoken while one of the two interlocutors was dragging.
} 
Table 3 Transcript and selected snapshots of Ana and Tammy's dragging while discussing a dynamic sketch related to the definition of a derivative

\begin{tabular}{|l|l|l|}
\hline Turn & What was said & What was done \\
\hline 1 & Tammy: From zero to positive, the slope is... & Tammy drags ' $x$ ' towards the right from the origin \\
\hline 2 & Ana: the tangent line is increasing. & \\
& Tammy: Tangent line is increasing. And from \\
& here to zero, it's decreasing. & \\
\hline 4 & Ana: And at zero, the tangent line is zero. \\
\hline 5 & Tammy: Zero. & \\
& &
\end{tabular}

To illustrate why I also consider the dragging actions to be gestures, it would be possible to imagine an environment where the dragging modality is not available. If a speaker moved his/her finger along a graph while referring to the tangent slope as "increasing" or "decreasing", this action could be considered a kind of gesture for communicating the idea, "as $x$ varies along this graph". In the present episode, the dynamic environment allowed the dragging with one finger on the touchscreen and the gesturing with the index finger to blend together as one action, into what I term the act of dragsturing $(\mathrm{Ng} 2014)$. The importance here is that the dragsturing is one action subsuming both dragging and gesturing characteristics - it both causes the point to be moved on the screen (dragging) and fulfills a communicational function (Sfard's definition of gestures). The present analysis is addressing the role of touchscreen-based DGEs for facilitating this form of communication.

Word use was also transformed in the presence of dragsturing. During the first exchange, Tammy used the phrases "is increasing" and "is decreasing" to describe the tangent slope, accompanied by dragsturing to communicate the change of tangent slope as the point was being dragged. This seemed to be mirrored immediately by Ana in the next turn. The use of the present continuous tense "is [verb]-ing" was a change from their previous discussion over a textbook diagram, where the students used the verb form "is [noun]" four times when discussing the same topic. Thus, in the present episode, dragsturing transformed the way Ana and Tammy communicated about the tangent slope. The verb forms suggest that "something is happening" at the very moment it is employed. This observation is made possible by a synchronic analysis of utterances, gestures and dragging in the students' mathematical discourse. 


\section{Other Gestures Facilitated by a Static Environment}

Both pairs of students used deictic gestures for pointing and gestures for conveying temporal relationships, as was noted in Ana and Tammy's communication. Besides these, two other interesting use of gestures were observed in Melissa and Yee's communication, when the two were discussing "linear approximation" in a static environment. At 06:47, Yee used his right index finger and thumb to form the shape of a "C" while he said, "and we get the values $r$, and actually the actual value is pretty close, right," with a high intonation (Fig. 4a). This is what I call a "measuring gesture" for conveying the distance or proximity between objects. Then, as he continued to speak, he brought the tips of his index and thumb together to touch each other, hence using another "measuring gesture". However, since the distance between the two points was "pretty close", the distance between his fingers was also reduced in his gesture (Fig. 4b).

Yee continued to use a combination of words and gestures to communicate the approximation of " $\Delta y$ " using " $d y$ ". In saying that " $d y$ and change of $y$ are a little bit different", he used his right pinky finger to point to the distance between the labelled lengths " $d y$ " and " $\Delta y$ " (Fig. 4c). This gesture resembled the kinds of deictic gestures that he had used before, but it was also different, in that it was the pinky finger that was used for pointing instead of the index finger. The change of finger for pointing suggested that Yee was communicating something slightly different here. More than just pointing, he may be thinking about a degree of precision with this gesture to complement his word use "a little bit different". The use of pinky was observed again when Yee talked about a different diagram, that of Newton's Method. With the use of his pinky for gesturing, he commented that, "the point is getting closer and closer" (11:30) when the Newton's Method was performed to approximate the root of an equation recursively.

It could be argued that both the gesture with a pinky finger and the "measuring gesture" were performed because it was not possible to move the objects close together in a textbook diagram physically; therefore, these gestures were used to convey the idea of bringing something close to another in the process of approximation. For example, in the diagram related to Newton's Method, the approximate roots $x_{1}, x_{2}, \ldots, x_{\mathrm{n}}$ were

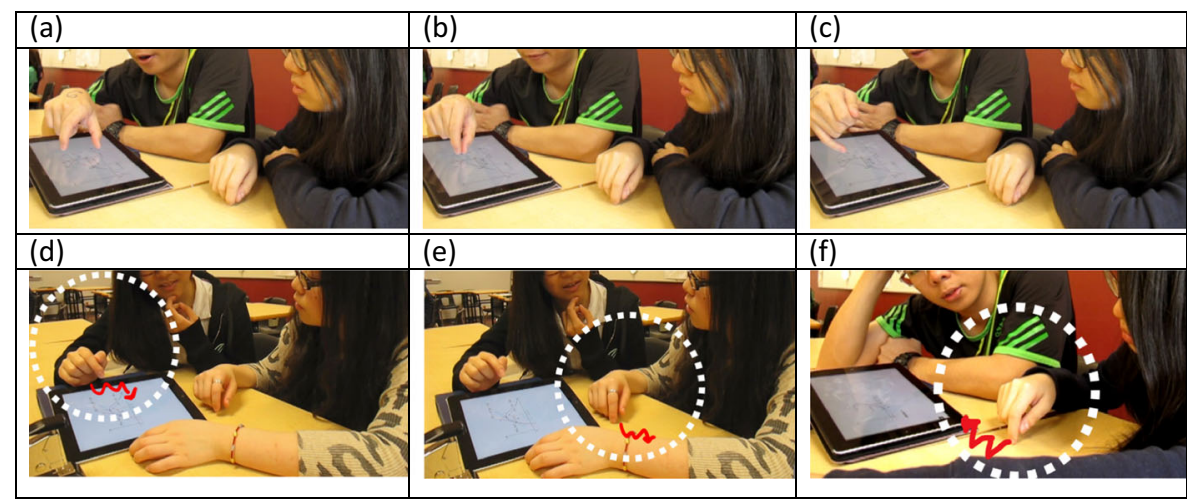

Fig. 4 a and b Yee's "measuring gesture"; c Yee's gesture with his pinky finger; "scribing gestures" used by (d) Ana, (e) Tammy and (f) Melissa 
explicitly shown in the textbook diagram. Hence, it could be difficult to express the action of obtaining $x_{n+1}$ recursively using the diagram. Perhaps the pinky finger was employed deictically (instead of the index finger) in order to convey a level of precision around approximating the root of an equation.

A little later, Melissa made a "scribing gesture" when she spoke, "As $x$ is approaching, $x$ approaching $x n$, and then like, the $x n$ is like closer to the $r$ " (12:05). I characterise the 'scribing gesture' as one where the finger enacts a pen, as if the gesturer was writing something down. These gestures were found in Ana and Tammy's discussion as well, sometimes accompanied by speech involving symbolic representations of calculus (Fig. 4d and e). The presence of scribing gestures was helpful for examining Melissa's thinking in the moment. In particular, her scribing gestures (Fig. 4f) suggested that she was thinking algebraically, perhaps about the Newton's Method formula, and not geometrically.

\section{Communicating "Change" in Two Environments}

In terms of the mathematical content communicated, it can be observed that the textbook diagram had occasioned certain ways talking about "change" for Melissa and Yee. Table 4 shows Yee's talk about change in a static and discrete sense when prompted by the given textbook diagrams. In terms of modal verbs, he said that "we can know" in two of his utterances, which implied that there were some concepts that the diagrams intended to convey. Occasionally, he also included the conditions of which the statements would hold true, such as, "So like when the graph of the function is decreasing, we can know that its derivative is less than zero. And when the graph of a function is increasing, the derivative is always greater than zero $(03: 10)$. The word "when" conveyed one static moment; moreover, the phrase "when [...] we can know" suggest that he was stating a calculus relationship as a timeless story.

Unlike Ana and Tammy, Melissa and Yee's discourse seldom contained deictic words, "this, that, here", etc. Without consistent usage of deictic words, the students' discourse lacked reference to the diagrams; rather, their discourse seemed to be focused on some mathematical concepts that existed outside of the diagrams. This was evident in the students' utterances and gestures as they discussed the diagrams. On three occasions, Yee discussed mathematics as if it existed outside of the diagrams with his mathematical-theorem-like talk.

Table 4 Yee's theorem-like discourse when discussing in static environment

(a) "So like when the graph of the function is decreasing, we can know that its derivative is less than zero. And when the graph of a function is increasing, the derivative is always greater than zero." (03:10)

(b) "Oh I got it... This is how to find the approximate value of a point by knowing one point and its derivative right? and its slope ... So from $\mathrm{P}$, we can know that the slope at $\mathrm{P}$, point $\mathrm{P}$ and we can find the function, and now we input another value which is $\mathrm{x}$ plus delta $\mathrm{x}$, and we get the values $\mathrm{r} . . . "$ (06:18)

(c) "I think it's mean value theorem ... ya should be mean value. So we have a function, you know a and b, and you draw a line across it and you get a secant, secant function, secant line function, and there must, if the function is continuous, so there must be a point $\mathrm{P}$, which its slope is equal to the slope of the secant line a, b. So for this here, at least one, it can be two, for Fig. 4, there is actually two points, P1 and P2, they both have the same slope as A, the secant line a, b. So there must be at least one point." (10:00) 
When discussing the textbook diagram related to "derivative functions", "linear approximation", "mean value theorem", Yee's talk resembled the genre of formal mathematics in the sense that he stated the givens statically and then deduced the results formally. One important characteristic of mathematical theorems is the absence of temporality. The majority of Yee's verb use, which was in the form of "is-noun" or "is-adjective," implied a timeless sense of calculus, as opposed to verb forms describing a process or dynamic relationships in calculus.

In addition, the different environments may have occasioned different ways of communicating "change". The analysis of the word "as", which presents dynamic qualities, supports this claim. Yee's first use of the word "as" was during the discussion of derivative functions in a static environment. Interestingly, he did not finish his sentence after beginning with the words, "so, as ..." (02:38). Rather, he self-repaired his speech and finished his sentence with the utterance: "from zero to $a$, from negative infinity to $a$, the graph is decreasing, right? So the graph of the derivative is under zero, less than zero ..." (02:40) In other words, Yee's sentence began with "as", but he immediately changed this way of talking about derivative functions by suggesting an interval for which the function is decreasing. Having begun with "as ...", he could have finished his sentence with something like, "as $\mathrm{x}$ increases, the tangent slope remains negative," which would convey continuous change, but he did not. Instead, he stated a property of the function, namely "the graph is decreasing" over the interval $(0,-\infty)$.

The notion that "the graph is decreasing" is discrete and static in nature, for it required that $\mathrm{f}(b)<\mathrm{f}(a)$ for all $a<b$. Although the verb itself ends in "-ing", a "decreasing" function does not necessarily imply a sense of motion or continuous change, as it is only necessary to provide an interval of which $\mathrm{f}(b)<\mathrm{f}(a)$. It could be further argued that the textbook diagram occasioned a discussion like this because it

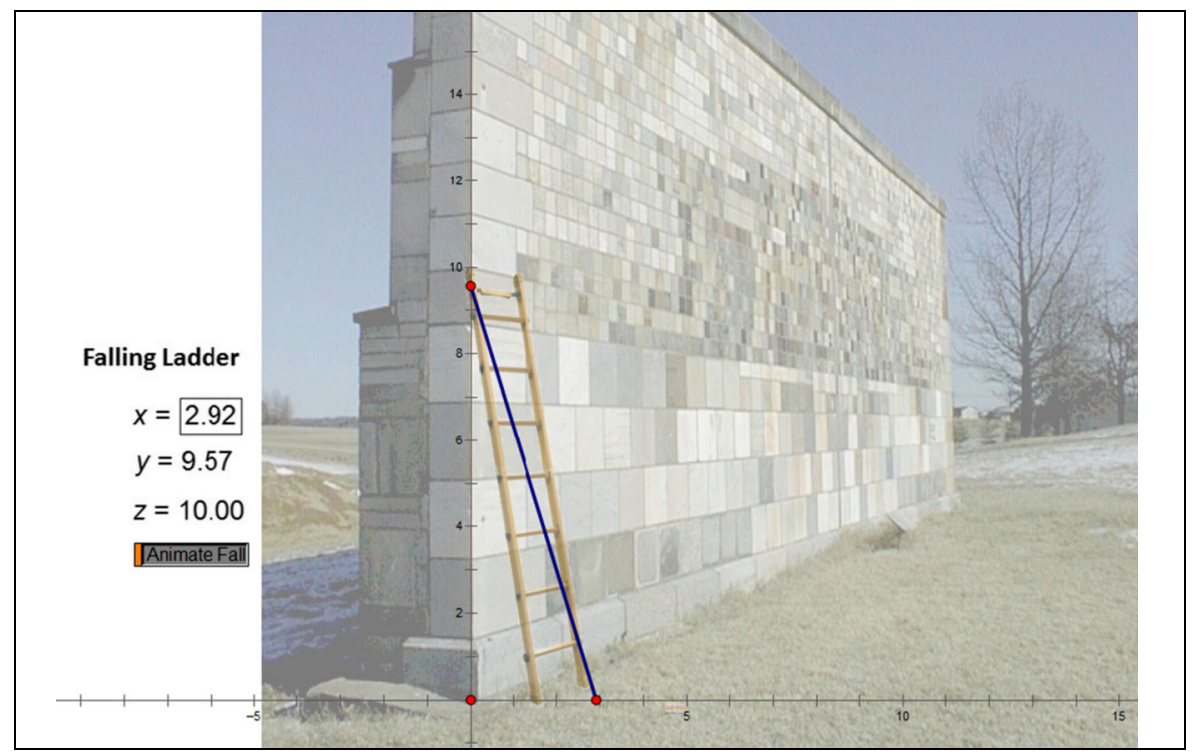

Fig. 5 Once the 'animate fall' button is pressed, the "ladder" as represented by the blue segment begins to 'fall' dynamically 
was showing a decreasing function statically. Had the dragging modality been available, it could have possibly occasioned a different kind of discourse, as I illustrate below.

In a dynamic environment, Yee did finish his sentence beginning with "as ...". When he pressed the action button to animate the falling of the ladder on the DGE screen (Fig. 5), he clearly communicated a sense of continuous change: "as the latter falls, we can see that $x \ldots$ is increasing and $y$ is decreasing, but $z$ remains constant" (17:10). By " $x \ldots$ is increasing and $y$ is decreasing", Yee was referring to the change of distance from the two ends of the ladder to the wall and ground respectively. The change communicated here was continuous and was visually mediated by the DGE.

Numerically, the change was conveyed by the values of $x, y$ and $z$ (rounded to 2 decimal place) shown in the sketch. Therefore, Yee might be addressing the continuous change of $x$ and $y$ both numerically and symbolically in his communication. Besides, Yee also communicated about the invariance of $z$. Although it may sound trivial to observe that $z$, the length of the ladder, was invariant, it was not trivial to comment on the invariance of $z$ as $x$ and $y$ are simultaneously changing as the ladder fell. This way of thinking about the change of variables simultaneously is necessary for the learning of related rates, which was the intended learning target of the sketch.

One example where Yee's word use conveyed discrete change was in his discussion of optimising area in a textbook diagram. As seen in Fig. 2a, the diagram consisted of three figures depicting the enclosure of a rectangular area that borders a river geometrically and numerically. Given the diagram, Yee did not communicate the variance of area and the dimensions of the enclosure in a dynamic and continuous sense; rather, he described a discrete change of the enclosed area. He used numerical values to reason why "you can't have a very long side" of enclosure (Table 5a). There was no indication of continuous change in his verb use.

In contrast, Yee used different words to describe the dimensions of the box that would optimise volume when the problem was posed in a dynamic sketch. He talked of the height of the box as "greater and greater" and the volume as getting "smaller and smaller" (Table 5b). Although the sketch showed numerical values of the dimensions (Fig. 6), he did not provide any numerical examples as he did with a textbook diagram. Moreover, functional dependency was also noted in his use of "if ..., then ..." statements: here, it was that the volume of the box was dependent on the height of

Table 5 Highlights of Yee's transcript when he discussed the optimisation of (a) area and (b) volume

(a) with textbook diagram

(b) with a DGE “[be]cause you can't have a very long one side and a very small two side, to maximize area. It has to be, both of them has to be like a big number, in order to have a bigger area. I think that's the point, like for this one both of them have 1200 that's pretty long, but the other two sides are only 100 , so the area is actually not that big right?" $\overline{(09: 30)}$

"If the length, the height of the box is greater and greater, the volume is smaller and smaller, you actually can see." (20:15)

"So now the height is decreasing, the length is increasing, the volume is actually getting bigger and bigger, but if the, if the length continues increasing, the volume decrease again, so there is one point like here... remains the greatest volume. I think that's it." $(21: 30)$ 


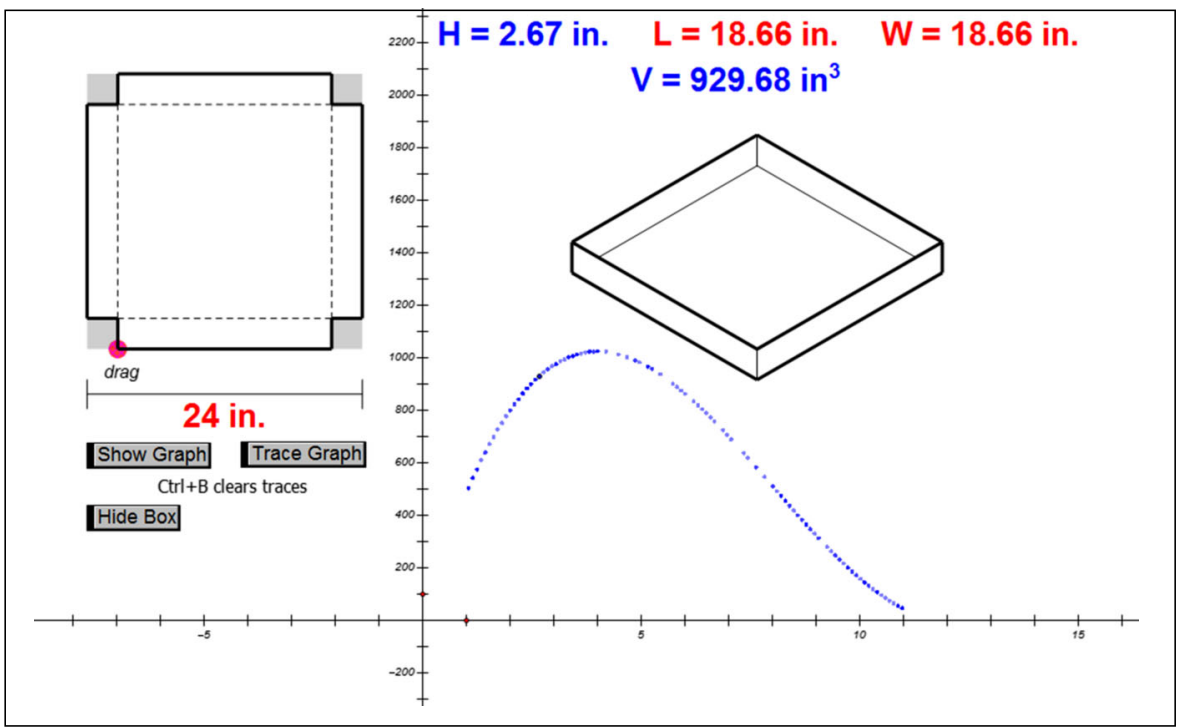

Fig. 6 A dynamic sketch conveying volume optimisation: the pink point on the $2 \mathrm{D}$ net of the box can be dragged to display different dimensions of the box 2- and 3-dimensionally, numerically and graphically

the box. As the height increases, the volume decreases. All of these sways of speaking supported the claim that Yee's discourse about continuous change was occasioned by a dynamic environment in the form of a DGE.

\section{Communicating Variance and Invariance Through Dragging}

Overall, the use of dragging was prevalent throughout both pairs of participants' discourse in a dynamic environment. It was used extensively as a routine to explore the dynamic relationship shown in the sketch. Figure 7 shows screenshots of some of Melissa and Yee's dragging routines: dragging with one finger (Fig. 7a), with two fingers (Fig. 7b) and two fingers one from each dragger (Fig. 7c). The consistent use of dragging changed the students' discourse about calculus: they did not use theorem-like talk to explain what the sketch was intended to say, but rather, communicated the variance, covariance and invariance that were implicated in the sketch through speech and dragsturing.

In some cases, dragsturing was used to explore the continuous change of variables after which the students described the relationships explored verbally. Conversely, in

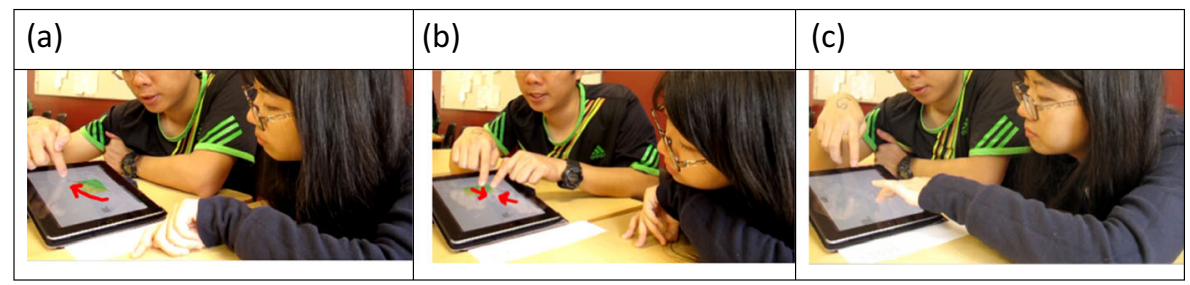

Fig. 7 Melissa and Yee's dragging routine with (a) one finger; (b) two fingers; (c) two different draggers, one finger each 
other cases, dragsturing was used to verify certain relationships after the students had initially hypothesised them. In either case, the dragging routine gave rise to verb forms that implied motion, such as "become" (14:13) and "getting closer and closer" (14:19). They also gave rise to exploring and verifying processes, none of which were observed when the students discussed the textbook diagrams.

In addition to exploring and verifying processes, the students also demonstrated a variety of other mathematical processes in a dynamic environment. Recall that the students were often previously stating static calculus ideas via theorem-like statements. In contrast, there were significantly less stating but more comparing and reasoning practices observed in the dynamic environment. For example, consider Yee's utterance:

If we have a linear function, the slope doesn't change. It remains the same because in this case $f x$ is three $x$ minus two and the derivative of $f$ is always three, it's a constant. (16:05).

The words "doesn't change" and "always" suggest that Yee had been observing the change of the derivative of the function $f(x)=3 x-2$. In order to say that the derivative is "always" three, one needs to compare the derivative of $f(x)$ across different values of $x$. This was achieved through Yee's dragging of $x$. Besides comparing, Yee was also reasoning about why "the slope doesn't change". As illustrated in his utterance, he was reasoning that the derivative was always three, a constant, hence the slope does not change.

Similarly, Melissa communicated the invariance of the tangent slope of $f(x)=3 x-2$, as follows:

If we drag the $x$, on the function of $x$, we get the tangent slope, and it's always $y$ equals three, and uhm here, the tangent line, the tangent line is the same as the $x$ values, and then the $y$-value is the secant, ah the tangent slope, so it's always three because it's on the same function, and the function has the same slope. (15:05)

What Melissa was communicating here was more than invariance of $f^{\prime}(x)$; she was communicating the covariance of two functions, $f(x)$ and $f^{\prime}(x)$. In the first part of her utterance, she was referring to "the function of $x$ ", and that its tangent slope was always equal to three. Then she mentioned that, "the tangent line is the same as the $x$-values", which I interpret as her comparing the $x$-values of the points $(x, f(x)),\left(x, f^{\prime}(x)\right)$, since they would be "the same". Finally, she suggested that the " $y$-value is the $[\ldots]$ tangent slope", which shows that she was attending to the mapping of $\left(x, f^{\prime}(x)\right)$ on $y=f^{\prime}(x)$. Hence, Melissa was referring to the simultaneous change in both $f(x)$ and $f^{\prime}(x)$ in this communication.

Related to variance and invariance was the idea of the general and particular. The contrast between Yee's discourse on the MVT across environments was illuminative in this respect. Recall that Yee's utterance resembled the genre of a mathematical theorem earlier and he used the phrase, "we can know that", which implied that there was some knowledge to be acquired cognitively from the textbook diagrams. With the DGE, however, Yee's discourse differed in several ways. First, he uttered "we can see that" as opposed to "we can know that". The transcript showed that Yee used the exact wording in the task of "we can see that" twice (both in the dynamic environment) and "we can know that" twice (both in the static environment). It was likely that the difference in 
verb use was due to the fact that dragging in DGE allowed one to "see" the change visually. This was significant because it shows that Yee's word use was connected with his dragging routine, and that he was making reference to the sketch directly. It was as if mathematics was happening right in front of him that he could "see", and it was no longer some knowledge to be acquired or to "know".

Secondly, Yee was able to use dragging to convey both generality and particularity in his communication. His dragging and word use complemented each other to convey generality, as he said, "we can choose random two points, $a, b$ in this case these two points, $a$ and $b$," while he dragged both a and $\mathrm{b}$ back and forth. In contrast, the idea of choosing two random points $a$ and $b$ was never communicated in a static environment. In fact, he used the verb "know" to introduce $a$ and $b$ in the textbook diagrams: "So we have a function, you know a and b". Later, Yee also dragged the point $c$ on the function to locate $x=c$ such that $f^{\prime}(\bar{c})$ would be equivalent to the secant slope through $f(a)$ and $f(b)$. His previous dragging of $a$ and $b$ to "choose two random" points, combined with the dragging of $c$ conveyed a sense of variance and invariance here, in that no matter which two points $a$ and $b$ he chose, he could find a point $c$ such that $f^{\prime}(c)=[f(b)-f(a)] /$ $[b-a]$. In other words, the theorem works for all chosen $a$ and $b$. Finally, he described a particular example of the MVT in the utterance, "which is 0.79 in this case right".

By illustrating particularity, he was also implying generality because he had said that the particular $a$ and $b$ were chosen randomly. This means that dragging was an important mode of communication about calculus and, more importantly, that calculus communication was situated in relation to the DGE. The textbook diagram occasioned Yee's talk about the MVT as a theorem (Table 6a), but the DGE occasioned Yee's talk about the MVT in terms of variance and invariance (Table 6b). The dynamism of the sketch and the draggable points $a$ and $b$ may well have facilitated this way of talking.

Table 6 Yee's talk about the MVT when given (a) a textbook diagram; (b) a DGE

(a) With textbook diagram

(b) With DGE

Defining the interval So we have a function, you know $\underline{a}$ and $b$, $(a, b)$

Drawing a secant line and you draw a line across it and you get a through $f(a)$ and $f(b)$ secant, secant function, secant line function, and there must,

Stating the conditions if the function is continuous, so there must and the results be a point $\mathrm{p}$, which its slope is equal to the slope of the secant line $a b$.

Verifying from the textbook diagram or dynamic sketch so for this here, at least one, it can be two, which is 0.79 in this case right? And that's for figure four, there is actually two points, $P 1$ and $P 2$, they both have the same slope as $A$, the secant line $a b$. So there must be at least one point. 


\section{Discussion and Conclusion}

The analysis provides strong evidence that the participants used different modes utterances, gestures and dragging - in their mathematical communication; and they communicated about the fundamental calculus ideas differently when prompted by different types of environments. Gestures were prevalent, but took on different communicative roles in different environments. In a static environment, the students mainly used utterances accompanied by deictic gestures and occasionally used gestures to communicate temporal relationships such as the "change of $x$ ". In addition, Yee changed the size of his "measuring gestures" to convey a change of distance, perhaps because he could not physically alter the objects on the textbook diagram. This communication routine evolved in the presence of the dragging modality and a dynamic environment. A new form of gesture emerged in the touchscreen dragging action with DGE and fulfilled the dual function of dragging and gesturing.

The frequent use of dragsturing in the analysis suggests that dragging was a significant communication routine for the students. They used dragsturing, accompanied by utterances, to talk about the variance of tangent slopes - and this was facilitated by the dynamic environment in the form of a DGE. The presence of dragsturing transformed the way calculus was communicated. As illustrated in the episode, Ana and Tammy resorted to verb forms that implied motion and temporality while they used dragging to change the tangent slope. This way of talking about calculus as a process was a change from their earlier discussions about the textbook diagrams, where the students used the "is [noun]" form to communicate a static sense of calculus ideas.

The students engaged in different mathematical processes in different environments. With the textbook diagrams, the students communicated about calculus procedurally and statically by naming the mathematical objects, by developing a formula and by communicating in a theorem-like discourse. The use of "scribing gestures" and utterances such as "we can know" and "we know $a$ and $b$ " provided evidence of their procedural and static calculus thinking. They communicated a discrete sense of change, complemented by the use of numerical examples.

With dynamic sketches, the students used dragsturing as a communication routine, accompanied by speech, to engage in exploring, verifying, comparing and reasoning processes. As a result, Yee's discourse changed from "we can know" to "we can see" when he began to drag on the touchscreen-based DGE. The touchscreen-dragging affordance may have facilitated this change of communication, by enabling Yee to "see" the dynamic relationships unfold in real time on the touchscreen DGE as he was dragging. The design of the sketch played an important role, since the draggable point was also the point of tangency on the function, which was a geometrical object. Thus, dragging the point has a dual meaning of changing the $x$-coordinate numerically, as well as physically moving the point of tangency geometrically. This design could potentially support students' communication of multiple representations in calculus, and it might have initiated dragsturing as the blending of dragging and gesturing the movement of the point of tangency.

Also, in line with Falcade et al. (2007), Melissa might have exploited the functionalities of the Dragging and Trace tools to communicate covariance of a function and its derivative function geometrically and dynamically. In summary, the dynamic environment, touchscreen technology, and the design of the sketch which include the 
exploitation of the dragging tool, all played a role in the students' discourse about dynamic features of calculus.

This study points to an expanded view of mathematical discourse that includes gestures, diagrams and dragging on touchscreen devices, in order to communicate dynamic aspects of mathematical ideas. Sfard's communicational framework - which defines gestures as communicational acts - was useful for understanding students' nonlinguistic communication such as gestures and dragging. Furthermore, this article extends Sfard's notion of visual mediators by distinguishing two kinds of visual mediation, dynamic and static. The distinction was important for this study because of the potential for the dynamic visual mediators such as gestures and DGEs to evoke temporal and mathematical relations (Ng and Sinclair 2013; Sinclair and Gol Tabaghi 2010), particular for the study of calculus (Núñez 2006). It also helped guide the analysis in terms of how "change" was conveyed in students' discourse, by distinguishing deictic gestures from gestures (or dragging) that conveyed temporal relationships.

This article presents the possibilities for extending the notion of visual mediators and routines to include gestures and dragging on touchscreen-based DGEs. As touchscreen learning technologies continue to enhance digital experience of learners, the blending of gestures and touchscreen-dragging for providing a haptic and mobile environment in mathematics learning is worthy of further examination (see also Sinclair and de Freitas 2014).

To revisit, this article has explored ways that may support student communication in calculus. The results of the study showed that different aspects of calculus communication can be facilitated and hindered with different media. First, in terms of fostering multiple representations in calculus, the study suggests that providing opportunities for students to learn with both textbook diagrams and dynamic sketches may help students develop their discourse about functions numerically, algebraically and geometrically. Second, the use of DGE was shown to be instrumental for facilitating dynamic and temporal - motion-based and action-oriented - thinking in calculus.

It is important to bear in mind that, at the time of study, the participants had already previously been taught the target calculus concepts with the use of touchscreen-based DGEs in their regular classrooms. Despite having learned the topics with the same dynamic sketches used in the study, the participants exemplified different discourses when prompted by two different types of environment. This offers important potential implications for classroom teaching, since it shows that mathematical thinking is not located in the heads but in the task and in the kinds of visual representations used (Chen and Herbst 2013). In order to develop and assess certain aspects of students' discourse in the study of functions and calculus, I have argued that providing situations for students to communicate these ideas in both static and dynamic environments, as well as adopting a multimodal view of communication, can be beneficial.

\section{References}

Arzarello, F. (2006). Semiosis as a multilingual process. Revista Latinoamericana de Investigación en matemática educativa, 9(special issue 1), 267-299. 
Arzarello, F. \& Paola, D. (2003). Mathematical objects and proofs within technological environments: an embodied analysis. In Proceedings of CERME-3, Bellaria, IT: CERME. (http:/www.mathematik.unidortmund.de/ erme/CERME3/Groups/TG9/TG9_Arzarello_cerme3.pdf).

Arzarello, F., Olivero, F., Paola, D., \& Robutti, O. (2002). A cognitive analysis of dragging practises in Cabri environments. ZDM - The International Journal on Mathematics Education, 34(3), 66-72.

Berry, J., \& Nyman, M. (2003). Promoting students' graphical understanding of the calculus. Journal of Mathematical Behavior, 22(4), 479-495.

Chen, C.-L., \& Herbst, P. (2013). The interplay among gestures, discourse, and diagrams in students' geometrical reasoning. Educational Studies in Mathematics, 83(2), 285-307.

Confrey, J., \& Smith, E. (1994). Exponential functions, rates of change, and the multiplicative unit. Educational Studies in Mathematics, 26(2/3), 135-164.

Cuoco, A. (1994). Multiple representations for functions. In J. Kaput \& E. Dubinsky (Eds.), Research issues in undergraduate mathematics learning (pp. 121-140). Washington: The Mathematical Association of America.

Falcade, R., Laborde, C., \& Mariotti, M. (2007). Approaching functions: Cabri tools as instruments of semiotic mediation. Educational Studies in Mathematics, 66(3), 317-333.

Ferrara, F., Pratt, D., \& Robutti, O. (2006). The role and uses of technologies for the teaching of algebra and calculus: Ideas discussed at PME over the last 30 years. In A. Gutiérrez \& P. Boero (Eds.), Handbook of research on the psychology of mathematics education: past, present and future (pp. 237-273). Rotterdam: Sense Publishers.

Graham, K. \& Ferrini-Mundy, J. (1989). An exploration of student understanding of central concepts in calculus. Paper presented at the Annual Meeting of the American Educational Research Association, San Francisco.

Hong, Y. \& Thomas, M. (2013). Graphical construction of a local perspective. In A. Lindmeier, \& A. Heinze (Eds.), Proceedings of the 37th Conference of the International Group for the Psychology of Mathematics Education (vol. 3, pp. 81-90). Kiel: PME.

Jackiw, N. (2001). The Geometer's Sketchpad. Emeryville: Key Curriculum Press.

Jackiw, N. (2011). SketchExplorer. Emeryville: Key Curriculum Press.

Lave, J., \& Wenger, E. (1991). Situated learning: legitimate peripheral participation. Cambridge: Cambridge University Press.

McNeil, D. (1992). Hand and mind: what gestures reveal about thought. Chicago: University of Chicago Press.

NCTM. (2000). Principles and standards for school mathematics. Reston: National Council of Teachers of Mathematics.

$\mathrm{Ng}$, O. (2014). The interplay between language, gestures, and dragging and diagrams in bilingual learners' mathematical communications. In P. Liljedahl, S. Oesterle, C. Nicol, D. Allan (Eds.), Proceedings of the Joint Meeting of PME 38 and PME-NA 36 (vol. 4, pp. 289-296). Vancouver: PME.

Ng, O. \& Sinclair, N. (2013). Gestures and temporality: Children's use of gestures on spatial transformation tasks. In A. Lindmeier, A. Heinze (Eds.), Proceedings of the 37th Conference of the International Group for the Psychology of Mathematics Education (vol. 3, pp. 361-368). Kiel: PME.

Núñez, R. (2006). Do real numbers really move? Language, thought, and gesture: the embodied cognitive foundations of mathematics. In R. Hersh (Ed.), 18 unconventional essays on the nature of mathematics (pp. 160-181). New York: Springer.

Robutti, O., \& Ferrara, F. (2002). Approaching algebra through motion experiments. In A. Cockburn, E. Nardi (Eds.), Proceedings of the 26th PME International Conference (vol. 1, pp. 111-118). Norwich, UK: PME.

Schwarz, B., \& Bruckheimer, M. (1990). The function concept with microcomputers: multiple strategies in problem solving. School Science and Mathematics, 90(7), 597-614.

Sfard, A. (2008). Thinking as communicating: Human development, the growth of discourses, and mathematizing. Cambridge: Cambridge University Press.

Sfard, A. (2009). What's all the fuss about gestures? A commentary. Educational Studies in Mathematics, 70(2), 191-200.

Sinclair, N., \& de Freitas, E. (2014). The haptic nature of gesture: rethinking gesture with new multitouch digital technologies. Gesture, 14(3), 351-374.

Sinclair, N., \& Gol Tabaghi, S. (2010). Drawing space: mathematicians' kinetic conceptions of eigenvectors. Educational Studies in Mathematics, 74(3), 223-240.

Sinclair, N., \& Yurita, V. (2008). To be or to become: how dynamic geometry changes discourse. Research in Mathematics Education, 10(2), 135-150.

Stewart, J. (2008). Calculus: early transcendentals (6th ed.). Belmont: Brooks Cole. 
Tall, D. (1980). Mathematical intuition, with special reference to limiting processes. In R. Karplus (Ed.), Proceedings of the Fourth International Conference for the Psychology of Mathematics Education (pp. 170-176). Berkeley: PME.

Tall, D. (1986). Building and testing a cognitive approach to the calculus using computer graphics (unpublished doctoral dissertation). Coventry: Mathematics Education Research Centre, University of Warwick.

Tall, D., \& Vinner, S. (1981). Concept image and concept definition in mathematics with particular reference to limits and continuity. Educational Studies in Mathematics, 12(2), 151-169.

Thompson, P. (1994). Images of rate and operational understanding of the fundamental theorem of calculus. Educational Studies in Mathematics, 26(2/3), 229-274.

Thompson, P., Byerley, C., \& Hatfield, N. (2013). A conceptual approach to calculus made possible by technology. Computers in the Schools, 30(1-2), 124-147.

Ubuz, B. (2007). Interpreting a graph and constructing its derivative graph: stability and change in students' conceptions. International Journal of Mathematics Education in Science and Technology, 38(5), 609637.

Vygotsky, L. (1978). Mind in society. Cambridge: Harvard University Press.

Weber, E., Tallman, M., Byerley, C., \& Thompson, P. (2012). Understanding the derivative through the calculus triangle. The Mathematics Teacher, 106(4), 274-278.

Wenger, E. (1998). Communities of practice: learning, meaning and identity. Cambridge: Cambridge University Press.

Williams, S. (1991). Models of limit held by college calculus students. Journal for Research in Mathematics Education, 22(3), 219-236.

Wittgenstein, L. (1953). Philosophical investigations. Oxford: Basil Blackwell.

Yerushalmy, M., \& Swidan, O. (2012). Signifying the accumulation graph in a dynamic and multirepresentation environment. Educational Studies in Mathematics, 80(3), 287-306. 